This is the accepted version of the following article: "J. Rousson, P. De Visschere, A. Vasiliev, A. Vetsuypens, and T. Kimpe. Front glass thickness requirements for high resolution polarized stereoscopic displays: A simulation platform. Journal of the Society for Information Display, 24(7):454-464, 2016." which has been published in final form at http://dx.doi.org/10.1002/jsid.456. This article may be used for non-commercial purposes in accordance with the Wiley Self-Archiving Policy http://olabout.wiley.com/WileyCDA/Section/id-828039.html.

\title{
Front glass thickness requirements for high resolution polarized stereoscopic displays: A simulation platform
}

\author{
Johanna Rousson *ł† Patrick De Visschere ${ }^{\ddagger}$ Anton Vasiliev * \\ Arnout Vetsuypens * Tom Kimpe*
}

August 10, 2016

\begin{abstract}
The tendency of the display market is towards displays with higher resolutions. Therefore, patterned retarder based stereoscopic displays require smaller front glass thickness to maintain good vertical viewing angle and limited crosstalk. To properly design these stereoscopic displays and quantify these requirements, we developed a simulation platform to predict radiance, polarization profile, and crosstalk over viewing angles and over wavelengths. Tunable parameters such as the distance between the pixels and the patterned retarder, and the optical properties of the patterned retarder are included. The simulation platform has been validated by comparing outcomes of simulations to measurements. We predict crosstalk accounting for both the human eye field of view and the diameter of the pupil. We found that to obtain a vertical viewing angle of at least $\pm 30^{\circ}$ and crosstalk of at most 0.11 for a display with a pixel pitch beyond $0.27 \mathrm{~mm}$, the display should include black absorbers and the thickness of the front glass should be at most $0.5 \mathrm{~mm}$. For higher resolution displays (pixel pitch no more than $0.21 \mathrm{~mm}$ ), a front glass thickness at most $0.15 \mathrm{~mm}$ is required to produce a vertical viewing angle beyond $\pm 14^{\circ}$ and a minimum viewing distance of $0.3 \mathrm{~m}$.
\end{abstract}

\section{Introduction}

The past years, efforts have been devoted to the development of displays providing a three-dimensional (3D) image to the observers [1]. The most widespread 3D-displays include technologies utilizing binocular disparity to generate depth in a scene. Amongst

\footnotetext{
${ }^{*}$ Healthcare division, Barco NV, Kortrijk, 8500, Belgium

${ }^{\dagger}$ email: johanna.rousson@barco.com

${ }^{\ddagger}$ ELIS, Ghent University, Ghent, 9000, Belgium
} 
them, autostereoscopic and stereoscopic technologies exist. Autostereoscopic displays do not require the observer to wear glasses, but increase of the viewing angle is at the expense of the image resolution. Stereoscopic displays have good viewing angle and allow for multiple observers. Patterned retarder technology is a widespread technology in stereoscopic displays for the consumer market. Especially, LG and Samsung are known to sell stereoscopic displays including a patterned retarder. An advantage of 3D-displays including a patterned retarder are the lightweight passive glasses. A typical patterned retarder based stereoscopic display is illustrated in Fig. 1. Pixels emit linearly polarized light (rays) which becomes either left- or right-handed circularly polarized depending on the row of the patterned retarder. To generate binocular disparity the odd rows of pixels are allocated to e.g. the left eye image and the even rows to the right eye image. The main cause of crosstalk in such systems is the distance between the patterned retarder and the pixel plane. In fact, since pixels do not emit collimated light, the larger the distance between the patterned retarder and the display, the more rays emitted by rows of pixels intended for retarder stripe $A$ will reach retarder stripe $B$.

Since the display market (both consumer [2,3] and medical [4]) is moving to displays with higher resolution, such as $4 \mathrm{~K}$, and more recently $8 \mathrm{~K}$, displays, it is of prime importance to adjust the distance between the patterned retarder and the pixels. Without a proper adjustment of this distance patterned retarder stereoscopic displays will not have such a large vertical viewing angle anymore. A few studies $[5,6,7]$ have been dedicated to the calculation of crosstalk for patterned retarder based 3D-displays. In [7] guidelines are given for designing the patterned retarder for a given 2D-display. In [5] and [6] a measure for the crosstalk is obtained using a simplified pixel geometry only. Our aim was to develop a more accurate simulation model using ray-tracing. Besides a more accurate pixel geometry it is then easy to take into account Fresnel reflections, the wavelength dependence of the refractive indices, as well as a more realistic model for the human eye. Therefore, we developed a simulation platform including all the relevant components of a display, i.e. the precise pixel structure (shape of the subpixels and the black matrix, the emission profile and emission spectrum), and the geometry and optical properties of the patterned retarder. With this platform we predict both polarization, radiance, and crosstalk profiles over viewing angles, and over wavelengths. We also assess crosstalk over viewing distance, and thus predict the minimum required viewing distance. Finally, both the field of view of the human eye [8] and the diameter of the pupil [9] are accounted for to measure crosstalk.

The details of the models included in the simulation platform and the framework used are given in section 2 . The validation of the platform, and an example of results obtained with the platform for two high resolution displays are described in sections 4 and 5 , respectively. Section 6 discusses the limits, but also, the potential extensions of the simulation platform. 


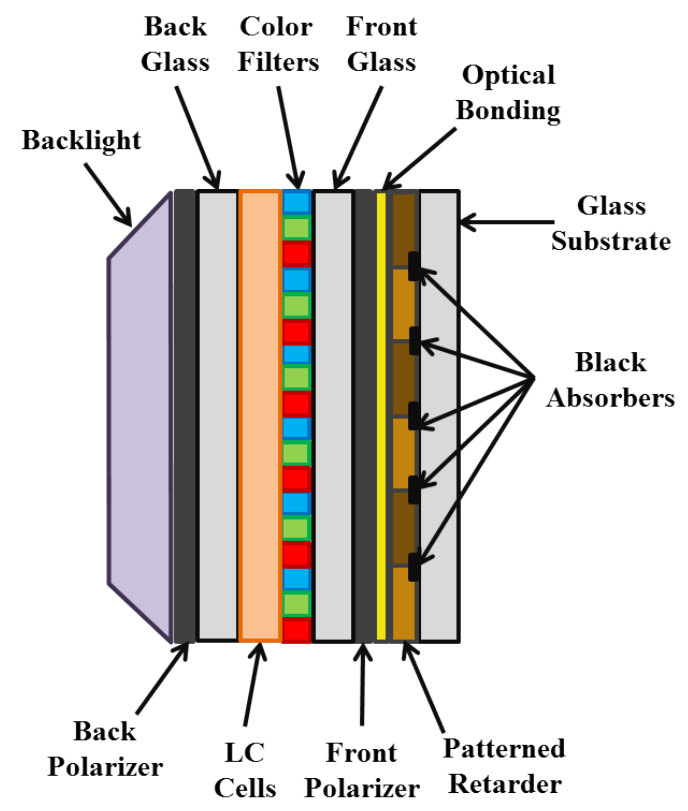

Figure 1: Schematic of the stereoscopic LC display $\left(\mathrm{LCD}_{1}\right.$ in section 3$)$ including a patterned retarder and black absorbers.

\section{Patterned retarder based stereoscopic display modeling}

Due to the optical properties of liquid crystals (LC) [10] and to the fact that LC cells are sandwiched between two crossed polarizers [11], light emitted by the LC panel is linearly (either horizontally or vertically) polarized. To generate a stereoscopic display a patterned retarder is mounted on the LC panel. As a consequence, the polarization state of the output light is spatially altered depending on the actual rows of pixels and the polarization state of the glasses worn by the observer. The patterned retarder consists of two quarter wave-plates with the axis oriented at $45^{\circ}$ or $135,^{\circ}$ measured w.r.t. the axis of polarization of the LC panel. Thus, left and right handed circular polarizations are generated for the odd and even rows of pixels, respectively. To ensure that the stereo images have a different polarization state, the left view is displayed on the odd rows of pixels while the right view is shown on the even rows of pixels. Finally, the spectacles worn by the observer contain left and right handed circular polarizers separating the two stereo images and sending them to the appropriate eye.

We developed a simulation platform to predict the radiance, the polarization, and the crosstalk behavior over viewing angles, and, over wavelengths, of a stereoscopic display including a patterned retarder. Since the geometry of the problem is complex (we have to find out which ray goes where depending on many parameters), we find ray-tracing as being the perfect solution for our simulation framework. Therefore, we modeled most objects separately from the others and include them into the ray-tracing software used. 
As illustrated in Fig. 1, the stereoscopic display can be decomposed into two main parts: (1) the 2D-display (i.e. the backlight, the back polarizer, the back glass, the TFT matrix, the LCs, the color filters, the front glass and the front polarizer), and (2) the patterned retarder. Additionally, black absorbers can be added on top of the patterned retarder to increase the vertical viewing angle. The pixels of the 2D-display and the patterned retarder are separated by the front glass, the front polarizer, and the optical bonding.

In the simulation platform, illustrated in Fig. 2, the distance between the patterned retarder and the pixels is defined as

$$
d_{3 D}=t_{\text {frontglass }}+t_{\text {frontpolarizer }}+t_{\text {bonding }},
$$

with $t_{\text {frontglass }}, t_{\text {frontpolarizer }}$, and $t_{\text {bonding }}$ the thicknesses of the front glass, the front polarizer, and the optical bonding, respectively. This simulation platform includes:

- The 2D-display (everything to the left of the front glass in Fig. 1) is modeled as a pixel plane with a pixel shape and an emission pattern over viewing angles (1. and 2. in Fig. 2). Therefore, the model applies equally well to a standard type display as to the COA-type.

- A single isotropic medium (3. in Fig. 2) embedding all the elements (the pixel plane, the front polarizer, the front glass, the patterned retarder, the optical bonding, the black absorbers) since we assume they all have the same refractive index $\mathrm{n}=1.5$.

- The front polarizer and the patterned retarder (4. and 6. in Fig. 2), which are treated as planes since their thicknesses are accounted for in $d_{3 D}$.

- The black absorbers, which can be present or not (7. in Fig. 2).

The simulation platform was developed with the optical design software Zemax. The models of the components were inserted mostly as Dynamic Link Libraries (DLL). All simulations described in this paper were run with one million rays in the non-sequential mode (i.e. rays can be split and scattered by the optical components).

In what follows we discuss these elements in more detail.

\section{$2.12 \mathrm{D}$ panel}

In the simulation platform the 2D LC panel is made of pixels (section 2.1.1) emitting light with a dedicated radiance profile over viewing angles, and of an O-type front polarizer (section 2.1.2) emitting linear horizontal polarization.

\subsubsection{Pixel structure and light distribution profile}

The more rays are emitted from the border of, e.g., the odd rows of pixels, the more rays are likely to reach the even rows of the patterned retarder. Therefore, crosstalk as well as the radiance of the display are expected to vary in function of the pixel structure. As a consequence, the precise structure of the subpixels is taken into account. In fact, depending on the selected 2D LC panel, the pixel structure (i.e. the shape of the emitting surface) varies, as illustrated in Fig. 3. 


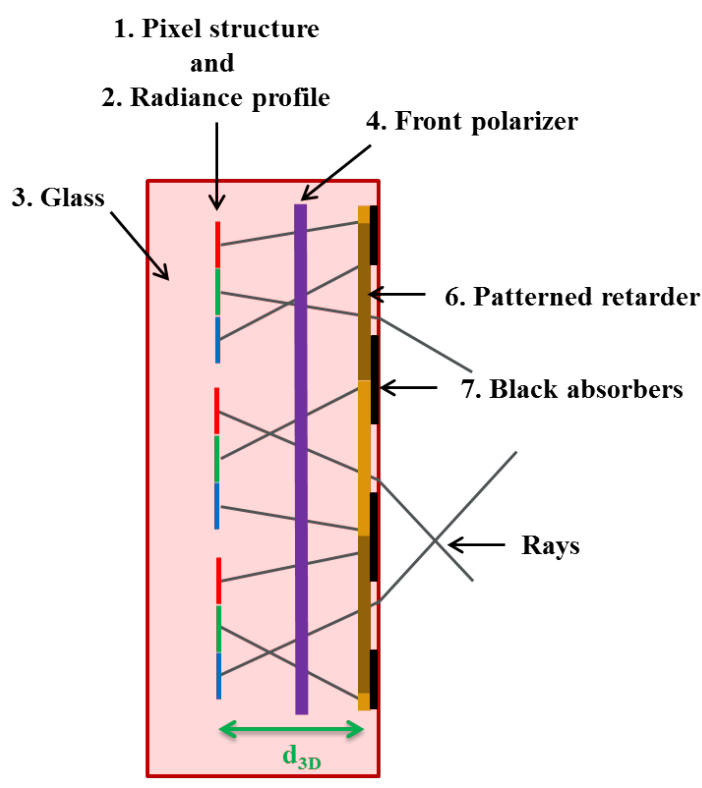

Figure 2: Schematic view of the simulation platform. $d_{3 D}$ is the distance between the patterned retarder and the pixel plane.

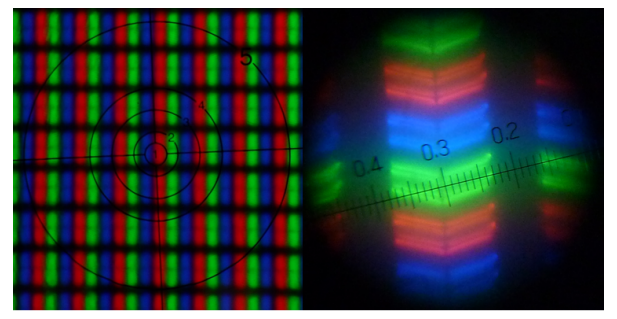

(a)

(b)

Figure 3: Examples of the pixel structure of two different displays, (a) a pixel composed of three subpixels and a fill factor of $35 \%\left(\mathrm{LCD}_{1}\right.$ in section 3$)$, and (b) a pixel made of three subpixels and a fill factor of $65 \%\left(\mathrm{LCD}_{2}\right.$ in section 3$)$.

The pixel structure is modeled by using data either provided by the LC panel manufacturer or has been measured with a microscope with a 10x objective lens. The light distribution over angles emitted by the 2D panel has been measured while displaying a uniform patch. Measurements of the different light profiles were carried out using either an EZContrastXL88MS multispectral device, or an EZContrast160D system, or a Microvision SS220 goniometer. Afterwards, the light profiles measured in air were corrected, using the Fresnel equations [12], to generate the profiles as if measured in glass. 


\subsubsection{Polarizer}

The front polarizer is modeled as a uniaxial anisotropic medium using the extended Jones matrix method (EJM) (i.e. a $2 \times 2$ matrix method) in the small birefringence approximation (i.e. no multiple reflections) as proposed by Yeh in [13]. The polarizer was assumed to be of O-type, i.e. only ordinary waves are transmitted. Consequently, the propagation matrix $P$ is

$$
P=\left(\begin{array}{ll}
1 & 0 \\
0 & 0
\end{array}\right)
$$

and the transfer matrix $M$ relating the transmitted wave amplitudes to the incident wave amplitudes can be written as:

$$
M=D_{\text {out }} P D_{\text {in }}
$$

where $D_{\text {out }}$ and $D_{\text {in }}$ are the output and input dynamical matrices $[13,14]$ representing the transmission and the reflection characteristics of each side of the interface, respectively. The ordinary index of refraction of the polarizer was set to $\mathrm{n}_{\mathrm{o}}=1.5$.

To verify the correctness of the polarizer model included in the simulation platform, a few simulations were carried out and compared with experimental data published in [13] and [15], which are based on the same model. Figure 4 depicts the transmission of the light through two crossed polarizers as published by Yeh in [13] and the results of the simulation using the aforementioned model of the polarizer. Over the five $\phi$ angles considered, the Root-Mean-Square Error (RMSE, Eq. 4) between our simulated transmission $T_{\text {simulated }}$ and the transmission calculated in [13] $T_{\text {calculated }}$ equals $6.77 \mathrm{x}$ $10^{-4}$.

$$
R M S E=\sqrt{\frac{\sum_{i=1}^{n}\left(T_{\text {simulated }, i}-T_{\text {calculated }, i}\right)^{2}}{n}}
$$

\subsection{Patterned retarder}

The retarder has also been modeled following the EJM method under the assumption of small birefringence $[13,16]$.

Two retarders overlaying the odd and even pixel rows respectively are included into the simulation platform. One retarder has a polarization axis making an angle $\phi$ of $45^{\circ}$ (see Fig. 5) with the (horizontal) $x$ direction, while $\phi=135^{\circ}$ for the other retarder. The pitch of the patterned retarder, as well as the optical properties of the retarders, i.e. the thickness and the birefringence, were obtained from the manufacturer of the patterned retarder. 


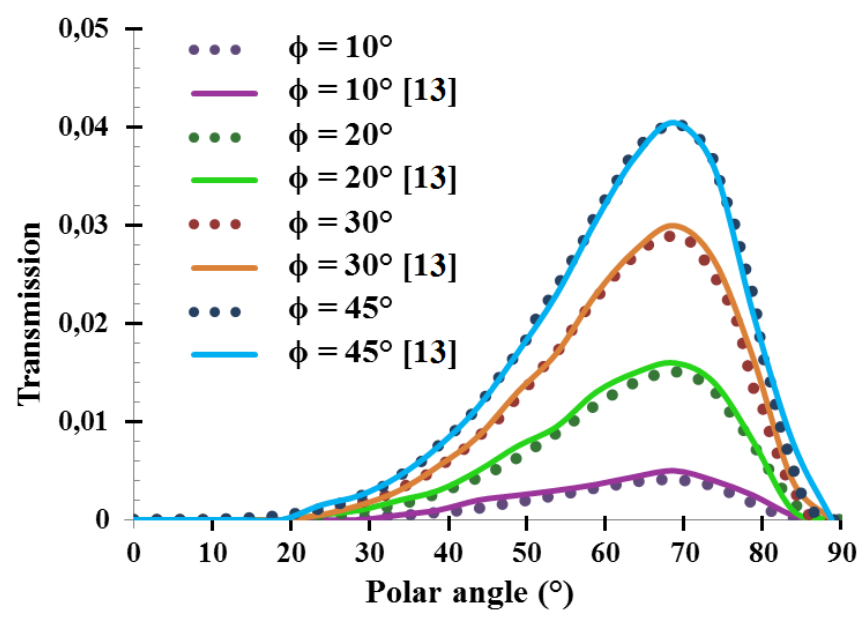

Figure 4: Measured and calculated transmittance for two crossed polarizers. Dotted lines were generated by the simulation platform. Solid lines were extracted from [13].

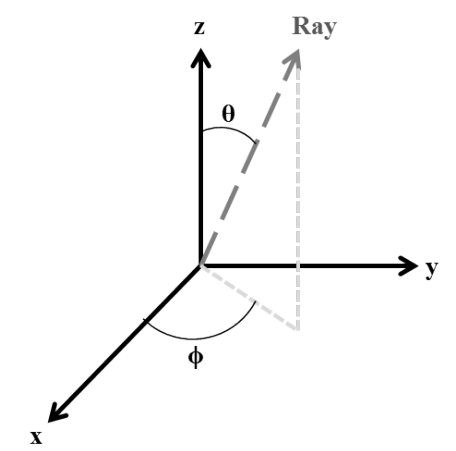

Figure 5: $(\mathrm{x}, \mathrm{y}, \mathrm{z})$ coordinate system with $\theta$ the polar angle and $\phi$ the azimuth.

\subsection{Polarization assessment}

The polarization profile over angles is studied using the Stokes vector given in [17] as:

$$
S=\left[\begin{array}{l}
S_{0} \\
S_{1} \\
S_{2} \\
S_{3}
\end{array}\right]
$$

with,

$$
\begin{aligned}
& S_{0}=P_{H}+P_{V} \\
& S_{1}=P_{H}-P_{V} \\
& S_{2}=P_{45}-P_{135} \\
& S_{3}=P_{R}-P_{L} .
\end{aligned}
$$


where $P_{H}, P_{V}, P_{45}, P_{135}, P_{R}$, and $P_{L}$ are obtained by measuring the radiance through a $0^{\circ}$ (i.e. horizontal) ideal (i.e. behaving the same regardless of the polar angle, and without considering any reflection) linear polarizer, a $90^{\circ}$ (i.e. vertical) ideal linear polarizer, a $45^{\circ}$ ideal linear polarizer, a $135^{\circ}$ ideal linear polarizer, a right-handed ideal circular polarizer, and a left-handed ideal circular polarizer, respectively. Therefore, the Stokes parameters are expressed in radiometric units, i.e. W.cm ${ }^{-2} \cdot \mathrm{sr}^{-1} S_{0}$ corresponds to the radiance of the actual display, and therefore, is proportional to the luminance of this display.

We compute the degree of linear polarization and the degree of circular polarization as:

$$
D O L P=\frac{\sqrt{S_{1}^{2}+S_{2}^{2}}}{\sqrt{S_{1}^{2}+S_{2}^{2}+S_{3}^{2}}}
$$

and

$$
D O C P=\frac{S_{3}}{\sqrt{S_{1}^{2}+S_{2}^{2}+S_{3}^{2}}},
$$

respectively. These quantities are used to compare the results of the simulation platform with the measurements.

\section{Experiments}

Two LCDs, both 2 Mpixel standard type TFT-LC displays with a pixel pitch of $0.27 \mathrm{~mm}$, denoted as $\mathrm{LCD}_{1}$ and $\mathrm{LCD}_{2}$ in the remaining of this paper, were both simulated and measured. The polarization profiles over angles were computed as given in Eqs. 7 and 8 . $\mathrm{LCD}_{1}$ and $\mathrm{LCD}_{2}$ have different values for $d_{3 D}$, respectivly, $d_{3 D}=0.88 \mathrm{~mm}$ and $d_{3 D}=0.705$ mm. Additionally, the radiance profiles over viewing angles and the pixel structures (see Fig. 3) of the 2D panels are different. Finally, unlike $\mathrm{LCD}_{2}, \mathrm{LCD}_{1}$ contains black absorbers.

To compare the simulated polarization profiles with the measurements, plots depicting 1D profiles of the polarization quantity (e.g. DOLP or DOCP) of the different outcomes are generated. A $1 \mathrm{D}$ profile of a polarization quantity is produced for a fixed azimuth $(\phi$, i.e., the projection of the actual ray on the $(x, y)$ in Fig. 5$)$ but varying polar angles $(\theta$, i.e., the angle between the actual ray and the z-axis in Fig. 5$)$.

All measurements related to $\mathrm{LCD}_{1}$ were carried out at Eldim (Hérouville-Saint-Clair, France) using an EZContrastXL88MS multispectral (31 band pass filters regularly distributed in the visible range $400 \mathrm{~nm}-700 \mathrm{~nm}$ ) measurement device at the central wavelength $\lambda=550 \mathrm{~nm}$ (i.e. the wavelength the patterned retarder is optimized for) [18]. With this device, measurements are taken for all viewing angles at once using Fourier optics. To measure polarization the EZContrastXL88MS device includes three linear polarizers $\left(0^{\circ}, 45^{\circ}\right.$, and $\left.90^{\circ}\right)$ and two wave-plates. $\mathrm{LCD}_{2}$ was measured using a Microvision SS220 goniometer [19] including mountable linear $\left(0^{\circ}, 45^{\circ}\right.$, and $\left.90^{\circ}\right)$ and right- and lefthanded circular polarizers [20]. Contrary to the Eldim device a separate measurement is performed for every viewing direction considered. The readings were subsequently corrected for the not $100 \%$ transmission of the polarizers. Both measurement devices have a radiance accuracy of $\pm 3 \%$. 


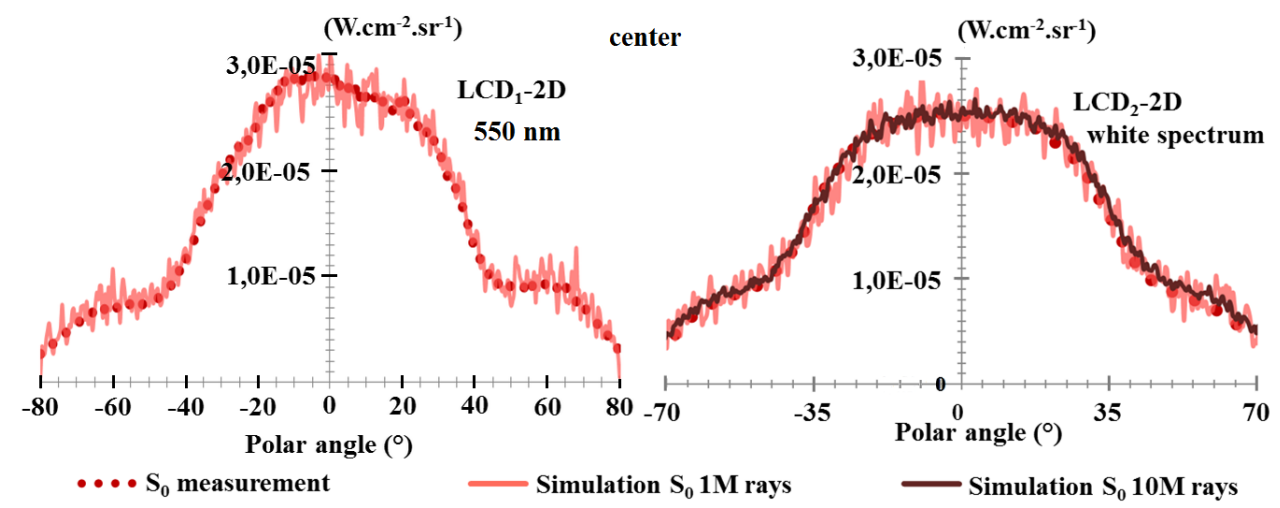

(a)

(b)

Figure 6: Measured and simulated Stokes parameter $\mathrm{S}_{0}$ as a function of the polar angle for an azimuth of $90^{\circ}$ for (a) $\mathrm{LCD}_{1}-2 \mathrm{D}$, and (b) $\mathrm{LCD}_{2}-2 \mathrm{D}$. Simulations were run with one million and ten million rays.

Both displays were analysed in 2D-mode (without patterned retarder) and in 3D-mode (with patterned retarder). Measurements were performed with halve (e.g. the odd lines) of the display fully on. In the simulations a sample of 12 (e.g. odd) rows of 40 pixels each was taken into account. Radiance and spectral measurements were done with the whole display fully on. Finally, except when otherwise mentioned, all measurements were performed at the center of the displays.

\section{Validation}

\subsection{D-display}

First, the simulation platform is validated for the 2D-displays only. Figures 6-a and 6-b depict 1D profiles of both the measured and the simulated $S_{0}$ (i.e. the radiance profiles) for an azimuth of $90^{\circ}$ for $\mathrm{LCD}_{1}-2 \mathrm{D}$ and $\mathrm{LCD}_{2}-2 \mathrm{D}$, respectively. As illustrated in Fig. 6-b, noise in the simulations can be attributed to the number of rays only.

Figures 7-a and 7-b depict 1D profiles of both the measured and the simulated DOLP and DOCP for an azimuth of $90^{\circ}$ for $\mathrm{LCD}_{1}-2 \mathrm{D}$ and $\mathrm{LCD}_{2}-2 \mathrm{D}$, respectively. The RMSE scores computed over polar angles for a $90^{\circ}$ azimuth between the simulated and the measured Stokes parameter $S_{0}$, DOLP, and DOCP are given in tables 1-a and 1-b for both displays. Whereas the measured and simulated $S_{0}$ and DOLP are very close together for both devices, there is a marked difference between the measured and simulated DOCP for $\mathrm{LCD}_{1}$. It should be noted that since $\mathrm{DOCP}^{2}+\mathrm{DOLP}^{2}=1$ and $\mathrm{DOLP} \approx 1$, the relative error on DOCP is much larger than on DOLP, and there is no contradiction between the DOLP and DOCP data. Assuming both 2D-displays are similar and considering the match for $\mathrm{LCD}_{2}$, we believe that the deviation for DOCP in Fig. 7-a must be attributed to retardation occurring in the EZContrastXL88MS measurement device. 


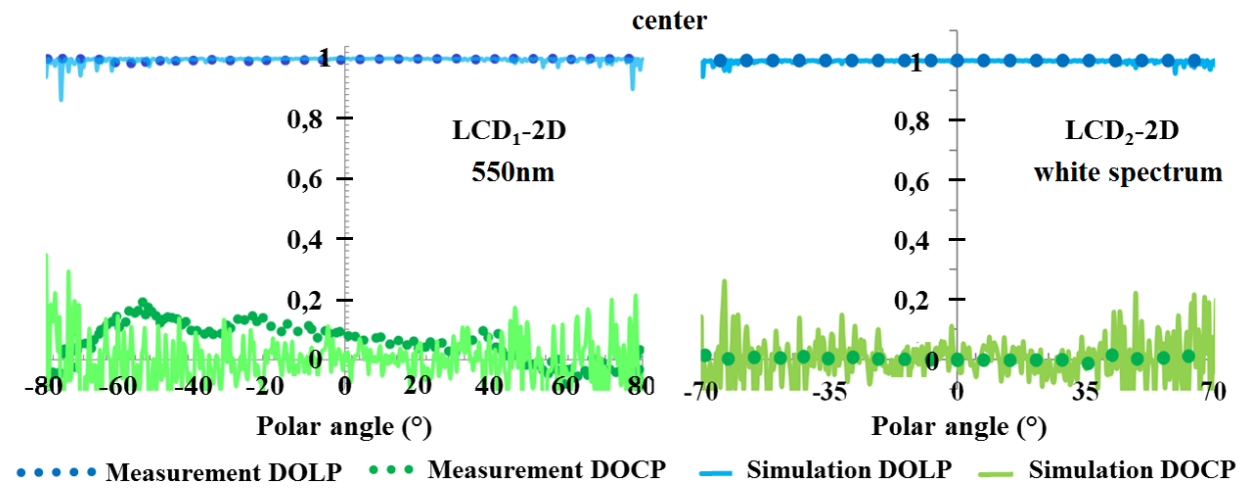

(a)

(b)

Figure 7: Measured and simulated DOLP and DOCP as a function of the polar angle for an azimuth of $90^{\circ}$ for (a) $\mathrm{LCD}_{1}-2 \mathrm{D}$, and (b) $\mathrm{LCD}_{2}-2 \mathrm{D}$.

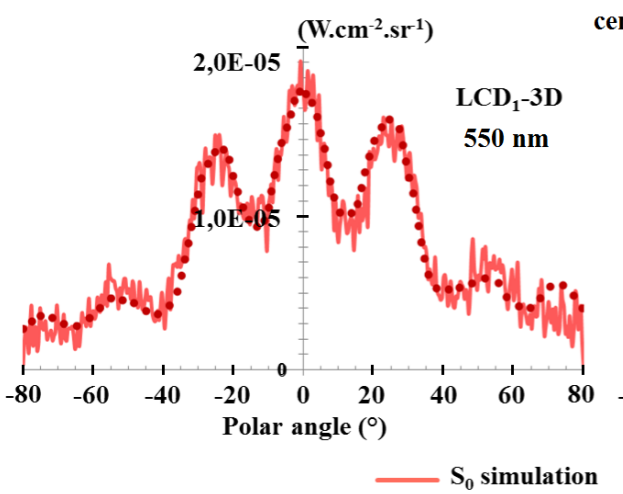

(a)

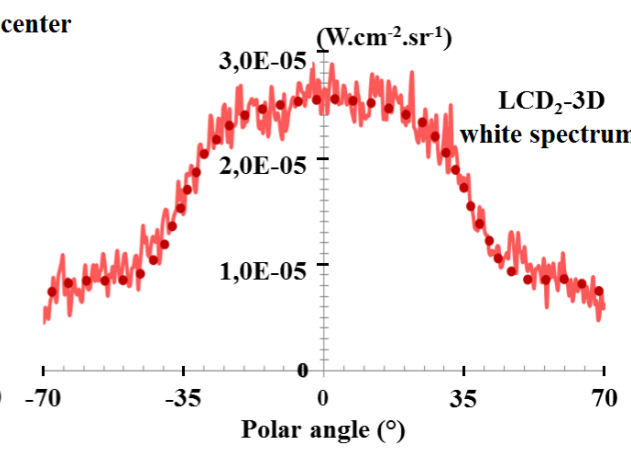

(b)

Figure 8: Measured and simulated Stokes parameter $\mathrm{S}_{0}$ as a function of the polar angle for an azimuth of $90^{\circ}$ for (a) $\mathrm{LCD}_{1}-3 \mathrm{D}$, and (b) $\mathrm{LCD}_{2}-3 \mathrm{D}$. Only the display on the left contains black absorbers.

\subsection{D-display}

As a second validation step, the simulated 3D-displays are compared with the measurements. Both LCDs have the same pixel pitch and the same patterned retarder pitch.

The Stokes parameter $S_{0}$ for both displays are shown in Fig. 8 for an azimuth of $90^{\circ}$. Again we find close agreement between the measurements and the simulations. The RMSE computed over polar angles amounts to $1.2 \times 10^{-6}$ and $1.53 \times 10^{-6}$ respectively (see table 1).

The comparison between the simulated and measured DOLP and DOCP are shown in Fig. 9 for $\mathrm{LCD}_{1}$ and in Fig. 10 for $\mathrm{LCD}_{2}$. This time and for the same reason as before, 


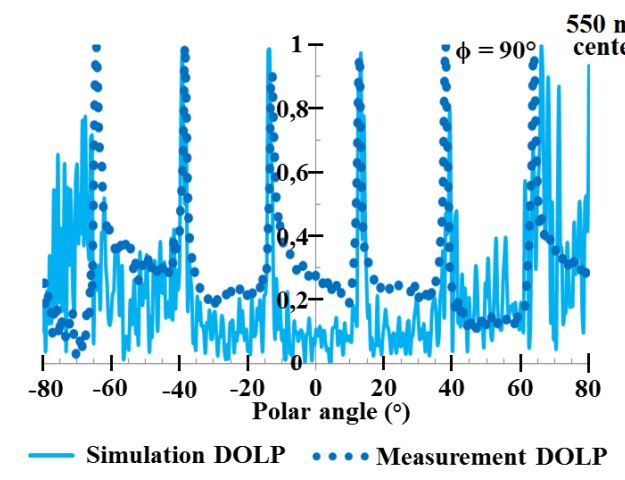

(a)

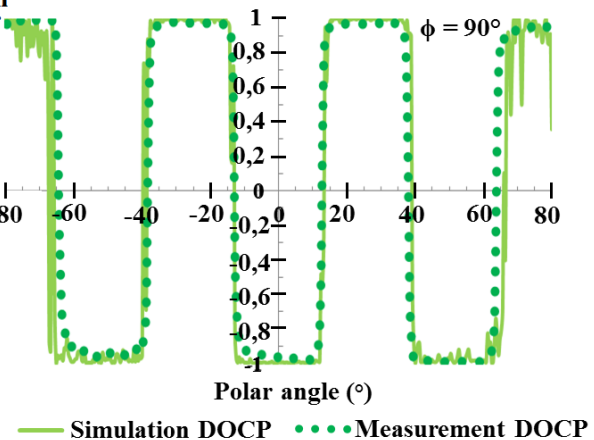

(b)

Figure 9: Measured and simulated (a) DOLP and (b) DOCP as a function of the polar angle for an azimuth of $90^{\circ}$ for $\mathrm{LCD}_{1}-3 \mathrm{D}$.

the discrepancies between simulations and measurements are most visible in the (small) DOLP, which can also be seen in the RMSE values listed in table 1.

Since $d_{3 D}$ is smaller for $\mathrm{LCD}_{2}$ than for $\mathrm{LCD}_{1}$, the plateaus of DOCP (i.e. the range of polar angles for which the light is almost perfectly circularly polarized) are slightly wider for $\mathrm{LCD}_{2}$ compared with $\mathrm{LCD}_{1}$.

The differences noticed between measured and simulated values of DOLP (and to a lesser degree of DOCP) can be attributed to thickness variations of the patterned retarder, whereas in the simulation a perfectly flat layer with the nominal $\lambda / 4$ thickness is assumed, and/or to variations of the orientation of the polarization axis.

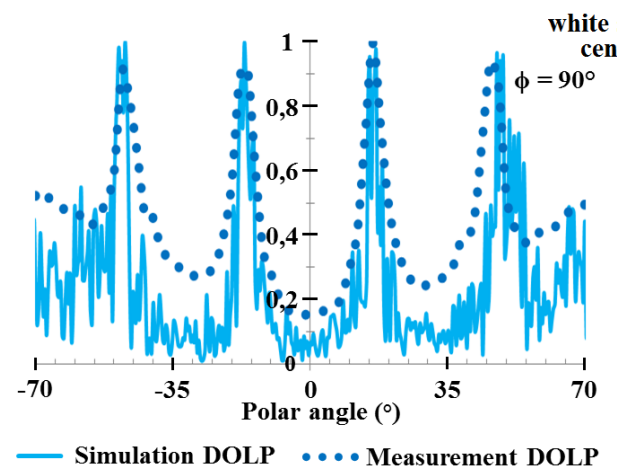

(a)

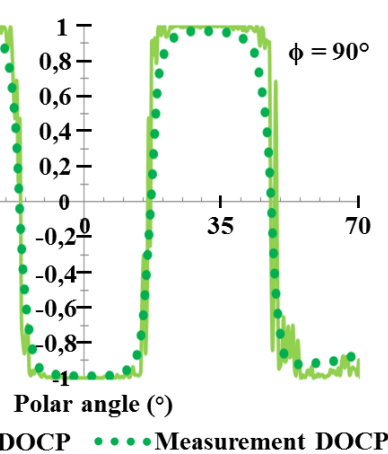

(b)

Figure 10: Measured and simulated (a) DOLP and (b) DOCP as a function of the polar angle for an azimuth of $90^{\circ}$ for $\mathrm{LCD}_{2}-3 \mathrm{D}$. 
Table 1: Overview of the Root-Mean-Square Error (RMSE) between measured and simulated Stokes parameter $\mathrm{S}_{0}$, DOLP and DOCP for $\mathrm{LCD}_{1}-2 \mathrm{D}, \mathrm{LCD}_{2}-2 \mathrm{D}, \mathrm{LCD}_{1}-3 \mathrm{D}$ and $\mathrm{LCD}_{2}-3 \mathrm{D}$. NS stands for not simulated.

Display Wavelength Position Azimuth RMSE

$\mathrm{S}_{0} \quad$ DOLP DOCP
(a) $\quad \mathrm{LCD}_{1}-2 \mathrm{D}$
$550 \mathrm{~nm}$
center
$90^{\circ}$
$1.28 \times 10^{-6} \quad 0.012$
0.135

(b) $\quad \mathrm{LCD}_{2}-2 \mathrm{D} \quad$ White spectrum

center

$90^{\circ}$

$1.34 \times 10^{-6} \quad 0.027$

0.079

(c) $\quad \mathrm{LCD}_{1}-3 \mathrm{D}$

$550 \mathrm{~nm}$

center

$90^{\circ}$

$1.20 \times 10^{-6} \quad 0.212$

0.122

(d) $\quad \mathrm{LCD}_{2}-3 \mathrm{D} \quad$ White spectrum

center

$90^{\circ}$

$1.53 \times 10^{-6}$

0.218

0.122

(e) $\quad \mathrm{LCD}_{1}-3 \mathrm{D}$

$550 \mathrm{~nm}$

top

$90^{\circ}$

NS

0.23

0.142

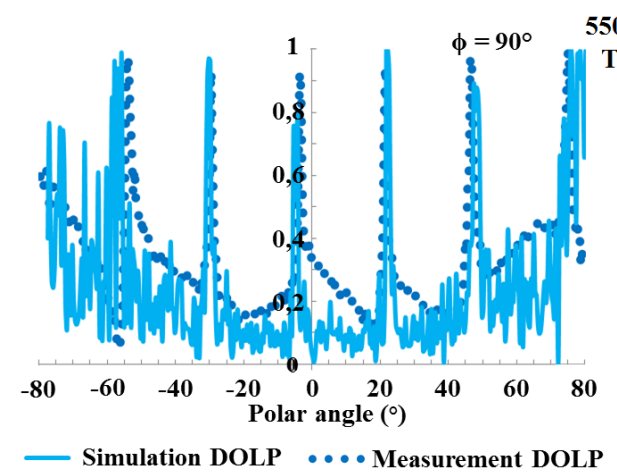

(a)

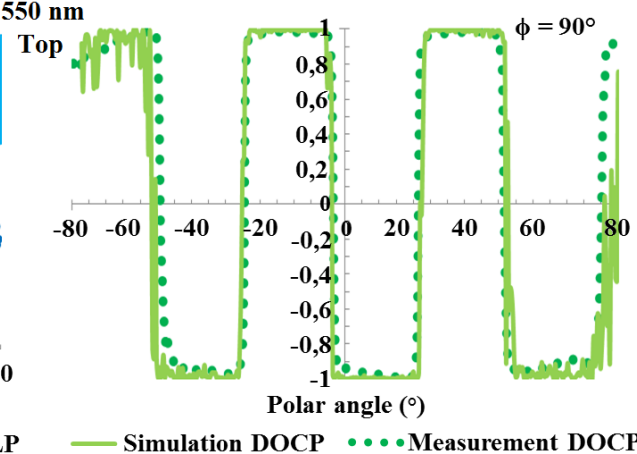

(b)

Figure 11: Measured and simulated (a) DOLP and (b) DOCP as a function of the polar angle for an azimuth of $90^{\circ}$ at $62 \mathrm{~mm}$ from the center of $\mathrm{LCD}_{1}-3 \mathrm{D}$ (next to the top of the display). 
On the other hand the alignment between the 2D-display pixels and the patterned retarder was found to be almost perfect. To check, we compared simulations and measurements at $62 \mathrm{~mm}$ from the center (i.e. center of the top half) of $\mathrm{LCD}_{1}-3 \mathrm{D}$ along the vertical crossing the center of the display. In the simulations the relative position of the pixel row and its retarder stripe was calculated from the pitch of the 2D-display and that of the patterned retarder and assuming they are properly aligned at the center of the display. Results given in table 1-e indicate that the discrepancies between simulations and measurements are slightly increased compared to the results at the center of the display (table 1-c). DOLPs and DOCPs curves, depicted in Figs. 11-a and 11-b, respectively, for an azimuth of $90^{\circ}$, suggest an angular deviation of $1^{\circ}$ to $1.5^{\circ}$ between measurements and simulations.

\subsection{Influence of the black absorbers}

As illustrated in Fig. 8 this simulation platform enables to predict the radiance profile over viewing angles for an arbitrary width of the simulated black absorbers. The black absorbers are evenly overlapping two rows of the patterned retarder and absorb all rays reaching them. A proper width of the black absorbers is crucial for the design of stereoscopic displays. To point out the influence of the black absorbers on the polarization profile, it is interesting to compare the degree of polarization (see Fig. 12), defined as

$$
\mathrm{DOP}=\frac{\sqrt{S_{1}^{2}+S_{2}^{2}+S_{3}^{2}}}{S_{0}},
$$

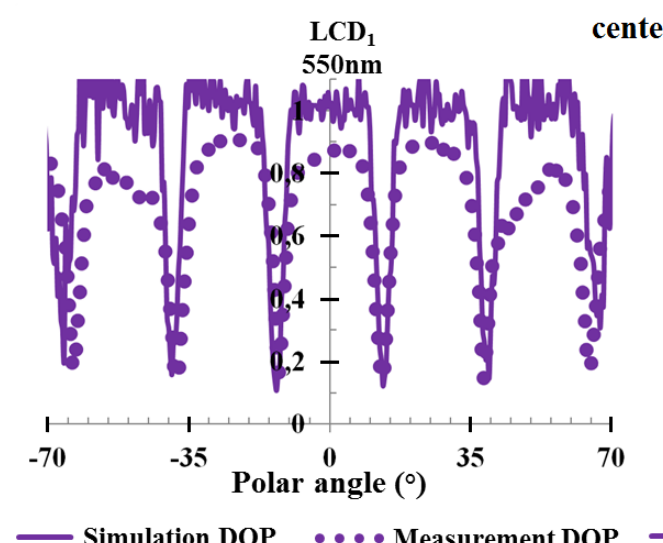

(a)

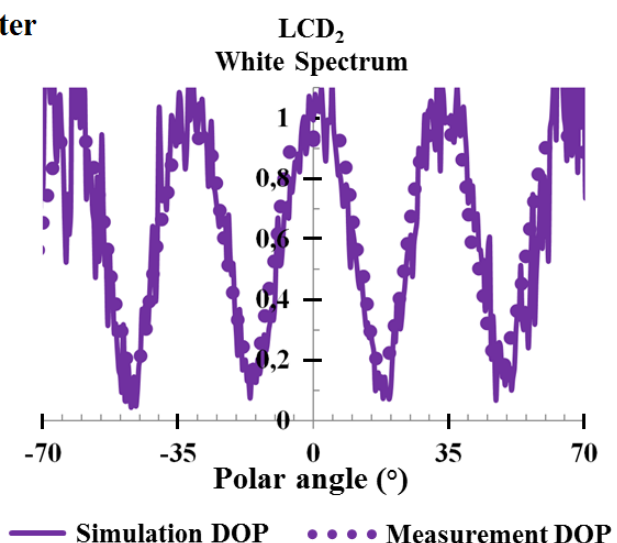

(b)

Figure 12: Measured and simulated DOP as a function of polar angle for an azimuth of $90^{\circ}$ for (a) $\mathrm{LCD}_{1}-3 \mathrm{D}$ and (b) $\mathrm{LCD}_{2}-3 \mathrm{D}$. For the latter the DOP has also been calculated for $550 \mathrm{~nm}$ resulting in the same triangularly shaped curve. Therefore the difference in spectra is not relevant. 
Although the data for $\mathrm{LCD}_{1}$ and $\mathrm{LCD}_{2}$ have been obtained for different spectra (pseudo monochromatic $550 \mathrm{~nm}$ versus a white spectrum) the difference between both types of display is due to the presence $\left(\mathrm{LCD}_{1}\right)$ or absence $\left(\mathrm{LCD}_{2}\right)$ of the black absorbers. Comparing Fig. 12 with Fig. 9 and Fig. 10 it is clear that the dips in DOP correspond with the transitions in DOCP, where the two uncorrelated types of polarization are maximally mixed. The black absorber limits the range of directions where both polarizations can mix as in Fig. 12-a. Without black absorber the DOP changes gradually and results in the triangular shaped curve in Fig. 12-b. Since the retarder is of 0-order, its depolarization effect [22, 23] can be neglected.

Therefore, black absorbers increase the vertical viewing angle where crosstalk must be limited. However, increasing vertical viewing angle is at the expense of the luminance (and the uniformity) of the display. Inserting black absorbers decreases the Stokes parameter $\mathrm{S}_{0}$ by $53 \%$ on average for vertical polar angles between $-20^{\circ}$ and $+20^{\circ}$ (see Fig. $6 \mathrm{a}$ and $8 \mathrm{a}$ ). Yet, the luminance is a key parameter for a display and it must not decrease below the luminance threshold of the given application.

\section{Application: crosstalk}

The aforementioned results indicate that the simulation platform enables to predict both the polarization and the radiance profiles over polar and azimuth angles, and over wavelengths of a stereoscopic display containing a patterned retarder. Therefore, the simulation platform can be used to assess crosstalk over viewing angles. We measure crosstalk $X$ as defined by the International Committee for Display Metrology in [21]. For instance, for the left eye, Crosstalk $X_{l}$ is expressed as

$$
X_{l}=\frac{L_{l} B W-L_{l} B B}{L_{l} W B-L_{l} B B},
$$

with $L_{l} B W$ the luminance behind the left glass for a white right side image and a black left side image, and vice versa for $L_{l} W B . L_{l} B B$ is the luminance behind the left glass when a black image is displayed. In the simulation $L_{l} B B=0$ and the left and the right glasses are made of a retarder followed by a linear polarizer. Simulations were performed for 522 odd rows of 500 pixels and a total of 270 million rays. The eye is modeled as a simple flat detector with an acceptance angle of $30^{\circ}$ (i.e. field of view that favors shape discrimination) and a diameter of $3 \mathrm{~mm}$ (i.e. the diameter of the pupil for a luminance of $250 \mathrm{~cd} / \mathrm{m}^{2}$, a field of view of $30^{\circ}$, and an observer aged $\left.30[9]\right)$.

In the consumer display market, 4K (4096 (W) x 2160 (H)) 31 inch and 55 inch displays are available. They correspond to pixel pitches $\simeq 0.17 \mathrm{~mm}$ and $0.30 \mathrm{~mm}$, respectively. In addition, in June 2015, LG demonstrated an 8K (7680 (W) x 4320 (H)) 98 inch display, with a pixel pitch $0.28 \mathrm{~mm}$. In October 2015, Japan Display released the prototype of an 8K 17.3 inch monitor, with a pixel pitch of about $0.05 \mathrm{~mm}$. Finally, in October 2014, Barco N.V. released a 12MP (4200 (W) x $2800(\mathrm{H})) 33.6$ inch diagnostic (medical) display, with a pixel pitch $\simeq 0.17 \mathrm{~mm}$. Therefore, we are interested in evaluating crosstalk as a function of $d_{3 D}$, for displays with pixel pitches ranging from $0.05 \mathrm{~mm}$ to $0.30 \mathrm{~mm}$. 
We selected three displays: (1) a 2MP (1920 (W) x $1200(\mathrm{H})) 24$ inch display with a pixel pitch $\simeq 0.27 \mathrm{~mm}\left(\mathrm{LCD}_{3}\right),(2)$ a $3 \mathrm{MP}(2048(\mathrm{~W}) \times 1536(\mathrm{H})) 21.3$ inch display with a pixel pitch $\simeq 0.21 \mathrm{~mm}\left(\mathrm{LCD}_{4}\right)$, and $(3)$ a $5 \mathrm{MP}(2560(\mathrm{~W}) \times 2048(\mathrm{H})) 21.3$ inch display with a pixel pitch $\simeq 0.16 \mathrm{~mm}\left(\mathrm{LCD}_{5}\right)$. In the simulation platform, $\mathrm{LCD}_{3}-3 \mathrm{D}$ and $\mathrm{LCD}_{5}-3 \mathrm{D}$ include the patterned retarder, and possibly black absorbers. $\mathrm{LCD}_{4}-3 \mathrm{D}$ only includes a patterned retarder.

In 2014, two studies [24, 25] reported that crosstalk is not perceivable up to 0.02 , but disturbing from 0.11 onwards. Adopting the 0.11 crosstalk constraint, we found that decreasing $d_{3 D}$ increases the vertical viewing angle. Therefore, stereoscopic displays with black absorbers and pixel pitch beyond $0.27 \mathrm{~mm}$ will have a vertical viewing angle of at least $\pm 30^{\circ}$ for $d_{3 D}$ smaller than $0.7 \mathrm{~mm}$ (i.e. front glass thickness up to $0.55 \mathrm{~mm}$ ), as depicted in table 2-(a). In addition, the minimum viewing distance can be as small as $0.5 \mathrm{~m}$. For higher resolution displays (pixel pitch no more than $0.21 \mathrm{~mm}$ ), $d_{3 D}$ of at most $0.1 \mathrm{~mm}$ is required to reach $\pm 25^{\circ}$ vertical viewing angle (table 2-(b)) if no black absorbers can be added. Vertical viewing angles of at most $\pm 15^{\circ}$ and $\pm 10^{\circ}$ (table 2-(c)) are obtained with $d_{3 D}=0.3 \mathrm{~mm}$ (i.e. front glass thickness up to $0.15 \mathrm{~mm}$ ) for pixel pitches up to $0.21 \mathrm{~mm}$ and $0.16 \mathrm{~mm}$, respectively. Nevertheless, the addition of black absorbers increases the viewing angle by $70 \%\left( \pm 10^{\circ}\right.$ to $\pm 17^{\circ}$, table 2 -(c) $)$ for a pixel pitch of $0.16 \mathrm{~mm}$ and $d_{3 D}=0.3 \mathrm{~mm}$. Finally, our simulations indicate that, properly configured, these displays can be used at viewing distances from $0.3 \mathrm{~m}$, which fit to the minimum required viewing distance of medical, and especially diagnostic, applications (where typical viewing distance ranges from $0.3 \mathrm{~m}$ to $0.6 \mathrm{~m}$ ). However, the minimum required viewing angle of high resolution medical displays (pixel pitch smaller than $0.21 \mathrm{~mm}$ ) typically being $\pm 40^{\circ}, d_{3 D}$ smaller than $0.2 \mathrm{~mm}$ (i.e. front glass thickness up to $0.05 \mathrm{~mm}$ ), and even $0.1 \mathrm{~mm}$, is required. Therefore, to realize patterned retarder based medical displays at least one of the constraints (viewing distance, viewing angle, crosstalk constraint, $d_{3 D}$ ) will have to be relaxed.

For $\mathrm{LCD}_{4}-3 \mathrm{D}$ we also assess crosstalk considering a pixel with an aperture of $100 \%$, and with $d_{3 D}=0.3 \mathrm{~mm}$ at a vertical viewing angle of $15^{\circ}$. We find crosstalk of $36 \%$ compared with $10 \%$ crosstalk found for the real pixel aperture.

Finally, modifying the acceptance angle changes crosstalk results. For example, at a vertical viewing angle of $15^{\circ}, \mathrm{LCD}_{4}-3 \mathrm{D}$ with $d_{3 D}=0.3 \mathrm{~mm}$ exhibits crosstalk of $20 \%, 10 \%$ and $13 \%$ for an acceptance angle of $5^{\circ}$ (i.e. typical narrow acceptance angle of measurement devices), $30^{\circ}$ (i.e. field of view of the human eye that favors shape discrimination) and $60^{\circ}$ (i.e. average total field of view of the human eye), respectively. These results indicate that to assess crosstalk, it might not be sufficient to take into account the rays along the viewing angle direction only, but that also the rays from the other directions should be taken into account. Differences found between $30^{\circ}$ and $60^{\circ}$ acceptance angles show that the maximum vertical viewing angle of these stereoscopic displays can slightly vary in-between observers. 


\section{Discussion and conclusions}

In both medical and consumer display markets the tendency is to build displays with smaller pixel pitches. However, in patterned retarder based stereoscopic displays, small pixels lead to smaller vertical viewing angles if the distance between the patterned retarder and the pixels is large. Therefore, we developed a simulation platform enabling to predict both polarization, radiance, and crosstalk profiles over polar and azimuth angles, and, over wavelengths. Crosstalk can also be measured as a function of viewing distance. The simulation platform is restricted to stereoscopic displays containing patterned retarders. The simulation platform enables to add a patterned retarder on top of existing 2D panels to evaluate their relevance for making a stereoscopic display. Additionally, parameters (e.g. the thickness of the front glass and the addition of black absorbers) can be tuned to assess the generated improvement. Finally, the accurate pixel geometry, the field of view of the human eye [8], and the diameter of the pupil [9] are accounted for when measuring crosstalk.

Table 2: Vertical viewing angle and minimum viewing distance for different distances separating the patterned retarder from the pixel plane $\left(d_{3 D}\right)$ for three different displays, accepting crosstalk of at most 0.11 .
Display
$d_{3 D}$
Black absorbers
Vertical viewing angle
Minimum viewing distance

$\begin{array}{rcccc} & 0.88 \mathrm{~mm} & \text { No } & \pm 3.5^{\circ} & 1.2 \mathrm{~m} \\ \text { (a) } \mathrm{LCD}_{3}-3 \mathrm{D} & 0.88 \mathrm{~mm} & \text { Yes } & \pm 14^{\circ} & 0.6 \mathrm{~m} \\ & 0.7 \mathrm{~mm} & \text { Yes } & \pm 30^{\circ} & 0.5 \mathrm{~m}\end{array}$

\begin{tabular}{|c|c|c|c|c|c|}
\hline \multirow{3}{*}{ (b) } & \multirow{3}{*}{$\mathrm{LCD}_{4}-3 \mathrm{D}$} & $0.3 \mathrm{~mm}$ & No & $\pm 15^{\circ}$ & $0.3 \mathrm{~m}$ \\
\hline & & $0.2 \mathrm{~mm}$ & No & $\pm 19^{\circ}$ & $0.3 \mathrm{~m}$ \\
\hline & & $0.1 \mathrm{~mm}$ & No & $\pm 25^{\circ}$ & $0.3 \mathrm{~m}$ \\
\hline & & $0.3 \mathrm{~mm}$ & No & $\pm 10^{\circ}$ & $0.3 \mathrm{~m}$ \\
\hline (c) & $\mathrm{LCD}_{5}-3 \mathrm{D}$ & $0.3 \mathrm{~mm}$ & Yes & $\pm 17^{\circ}$ & $0.3 \mathrm{~m}$ \\
\hline
\end{tabular}


We found that, to generate vertical viewing angles of at least $\pm 30^{\circ}$ (with a crosstalk constraint of 0.11), displays with pixel pitch beyond $0.27 \mathrm{~mm}$ should include black absorbers and a front glass with a thickness of at most $0.5 \mathrm{~mm}$. For higher resolution displays (pixel pitch no more than $0.21 \mathrm{~mm}$ ), a front glass thickness up to $0.15 \mathrm{~mm}$ is required to produce a vertical viewing angle of at least $\pm 15^{\circ}$. Finally, properly configured high resolution polarized stereoscopic displays can be used for viewing distances as small as $0.3 \mathrm{~m}$, which fit to the viewing distance requirement of medical, and especially, diagnostic, applications. However, to allow for very large viewing angles (typically, beyond $40^{\circ}$ in medical applications), at least one of the constraints (viewing distance, crosstalk constraint, $d_{3 D}$ ) will have to be relaxed.

\section{Acknowledgements}

This work has been supported by the IWT (Institute for the Promotion of Innovation by Science and Technology in Flanders) in the context of the Baekeland Grant "Optical simulation, modeling and evaluation of 3D medical displays," 110542.

\section{References}

[1] N. S. Holliman and N. A. Dodgson and G. E. Favalora and L. Pockett, "ThreeDimensional Displays: A Review and Applications Analysis," IEEE transactions on broadcasting $\mathbf{5 7}(2), 362-371(2011)$.

[2] J. Gulick, "LG Shows Off $8 \mathrm{~K}$ Display And Curved-Edge Galaxy Note Edge Competitor," http://hothardware.com/news/ lg-display-shows-off-8k-display-and-phone-with-curved-edges, 2015 (December 17th 2015).

[3] Japan Display Inc., "JDI Announces Development of World's First Standard Monitor Size 17.3-inch 8K4K LCD Module," http://www.j-display.com/english/news/ 2015/20151001.html, 2015 (December 17th 2015).

[4] Barco N.V., "Join the revolution in the radiology reading room," https://www.barco.com/en/News/Post/2014/10/16/ Join-the-revolution-in-the-radiology-reading-room, 2014 (December 17th 2015).

[5] H. Hong and J. Jang and D. Lee and M. Lim and H. Shin, "Analysis of angular dependence of 3-D technology using polarized eyeglasses," Journal of the Society for Information Display 18(1), 8-12 (2010).

[6] C. Ma and Y. Chang, "A Simulation Platform and Crosstalk Analysis for Patterned Retarder 3D Display," SID Symposium Digest of Technical papers 42(1), 808-811 (2011).

[7] T. Tanabe and T. Sato and K. Fukaishi and Y. Kakubari, "Circularly Polarized (CPL) 3D Monitors Attract Attention Again for Medical Applications," SID Symposium Digest of Technical papers 46(1), 987-990 (2015). 
[8] J. M. Pellerito, "Assessments in driver rehabilitation," Handbook of Assessment in Clinical Gerontology, P. A. Lichtenberg, ed. (Academic Press, 2010).

[9] A. B. Watson and J. I. Yellott, "A unified formula for light-adapted pupil size," Journal of Vision 12(10), 1-16 (2012).

[10] P. Yeh and C. Gu, Optics of Liquid Crystal Displays (John Wiley \& Sons, 2010).

[11] R. H. Chen, Liquid Crystal Displays: Fundamental Physics and Technology (John Wiley \& Sons, 2011).

[12] J. M. Bennett, "Polarization," in Handbook of Optics: Volume I, M. Bass, ed. (Mc Graw-Hill, 1995).

[13] P. Yeh, "Extended Jones matrix method," J. Opt. Soc. Am. 72(4), 507-513 (1982).

[14] P. Yeh, "Optics of anisotropic layered media: a new 4x4 matrix algebra," Surface Science 96, 41-53 (1980).

[15] G. Hass and H. Wöhler and M. Fritsch and D. A. Mlynski, "Polarizer model for liquid-crystal devices," J. Opt. Soc. Am.5(9), 1571-1575 (1988).

[16] P. Yeh, "Extended Jones matrix method. II," J. Opt. Soc. Am.10(5), 966-973 (1993).

[17] R. A. Chipman, "Polarimetry," in Handbook of Optics: Volume II, M. Bass, ed. (Mc Graw-Hill, 1995).

[18] Eldim, "EZContrast MS Serie," http://www.eldim.fr/index.php?option=com_ zoo\&task=item\&item_id=92\&Itemid=471, 2016 (February 2nd 2016).

[19] Microvision, "SS220 Flat Panel Analysis System," http://www.bandp.co.kr/ electronic/pdf/220_Data_sheet.pdf, 2003 (February 2nd 2016).

[20] Edmund Optics, " $25.4 \mathrm{~mm}$ Dia. Left-Handed Plastic Circular Polarizer," http://www.edmundoptics.eu/optics/polarizers/circular-polarizers/ circular-polarizers/88098/, 2015 (February 2nd 2016).

[21] SID and ICDM, "3D \& Stereoscopic Displays," in Information Display Measurements Standard version 1.03, ICDM (2012).

[22] A. Domanski. Polarization degree fading during propagation of partially coherent light through retarders. Opto-Electronics Review, 13(2):171-176, 2005.

[23] S. ling Lu and A. P. Loeber. Depolarization of white light by a birefringent crystal. J. Opt. Soc. Am., 65(3):248-251, Mar 1975.

[24] J. Rousson and J. Couturou and A. Vetsuypens and L. Platisa and A. Kumcu and T. Kimpe and P. Philips, "Subjective quality and depth assessment in stereoscopic viewing of volume-rendered medical images," Proc. SPIE 9011, (2014).

[25] K. Wang and B. Andrén and M. Hussain and K. Brunnström and J. Osterman, "Perception and annoyance of crosstalk in stereoscopic 3D projector systems," Proc. SPIE 9011, (2014). 